\title{
Transdermal Patches For Delivery of Beta-Blockers
}

Received 15 June, 2021, accepted 17 June, 2021

Abstract Transdermal matrices containing $1.258 \mathrm{mg} / \mathrm{cm}^{2}$ of propranolol and consisting of ethylcellulose (EC), castor oil, and hydroxypropylmethylcellulose (HPMC) or halloysite (HA) were prepared. They were evaluated by tests such as folding endurance, moisture content and absorption, and paddle dissolution test. Of the total amount of propranolol in the samples ( $20 \mathrm{mg}), 28.41 \%$ $\pm 3.30 \%$ was released from the EC film after 24 hours, the addition of HA $20.94 \% \pm 1.52 \%\left(f_{1}=61.82 \pm 7.70, f_{2}=53.61 \pm 4.25\right)$ or HPMC $36.05 \% \pm 6.18 \%\left(f_{1}=34.48 \pm 8.79, f_{2}=65.02 \pm 5.33\right)$. The dissolution profiles of HA and HPMC films were compared with each other $\left(f_{1}=51.35 \pm 12.56, f_{2}=59.20 \pm 9.43\right)$.

Keywords ethylcellulose-halloysite-hypromellose-propranolol-transdermal therapeutic system

\section{INTRODUCTION}

Beta-blockers are one of the most used molecules in the therapy of cardiovascular diseases. They are mainly formulated as conventional dosage forms (Aquil et al., 2006). Management of cardiovascular diseases often requires, besides a change of lifestyle, also a long-term pharmacotherapy. This kind of therapy can lead to insufficient patient compliance because of dosage frequency. Orally administered beta-blockers are characterised by the need for doses that are more frequent, significant first-pass effects and variable bioavailability. Transdermal patches prolong the time interval of therapeutic plasma levels, which decreases the incidence of side effects and the dosage frequency. Transdermally administered drugs avoid the hepatic first-pass effect, which increases their bioavailability. Another advantage of transdermal patches is their easy application, which can also contribute to higher patient compliance (Ahad et al., 2015).

Model drug propranolol is significantly hepatically metabolised, which causes low bioavailability 30\%-35\% after oral administration. Its elimination half-life is 2-6 hours and the partition coefficient $\log \mathrm{P}$ is 3.03 (Calatayud-Pascual et al., 2018). Thanks to these properties, propranolol is suitable for transdermal administration.

\section{METHODS}

Chemicals: Propranolol hydrochloride was purchased from Fagron, a.s. (Olomouc, Czech Republic), ethylcellulose (EC) from Acros Organics (New Jersey, PA, USA), hydroxypropylmethylcellulose (HPMC) from Dr. Kulich Pharma, s.r.o (Hradec Králové, Czech Republic) and halloysite (HA) nanoclay from Aldrich Chemistry (Saint-Louis, MO, USA). Film preparation was carried out by solvent casting method. Composition of the films is shown in Table 1. The dispersions were prepared by mixing EC in ethanol with castor oil as a plasticiser for 5 minutes at $750 \mathrm{rpm}$. Propranolol was dissolved in ethanol and added to the dispersion. Either HPMC or HA was added to the prepared dispersions and the mixing was continued for 15 minutes at 750 rpm. Films were poured into Petri dishes previously wiped out with glycerine. HA-containing films underwent incorporation of the drug in a vacuum, which was applied 3 times for 5 minutes. Films were dried (UNB400; Memmert, Schwabach, Germany) after 12 hours at $60^{\circ} \mathrm{C}$ and covered with funnels.

Folding endurance was determined by repeatedly folding the film at the same place until visible damage was caused. The number of times the films could be folded without breaking gives the folding endurance value. We considered 
Table 1. Composition of formulations (in grams).

\begin{tabular}{|c|c|c|c|c|c|c|}
\hline Film & Propranolol & Ethanol & Castor oil & Ethylcellulose & Halloysite & Hypromellose \\
\hline $\mathrm{E}$ & 0.02 & 10 & 0.162 & 0.36 & - & - \\
\hline Ha1 & 0.02 & 10 & 0.162 & 0.36 & 0.0225 & - \\
\hline Ha2 & 0.02 & 10 & 0.162 & 0.36 & 0.0450 & - \\
\hline HPMC1 & 0.02 & 10 & 0.162 & 0.36 & & 0.0225 \\
\hline HPMC2 & 0.02 & 10 & 0.162 & 0.36 & & 0.0450 \\
\hline
\end{tabular}

the film as favourable if it lasted without obvious damage after a hundred folds (Sanap et al., 2008).

Percentage moisture content was calculated using Equation (1), where $m_{1}$ is the initial weight of a film and $m_{2}$ is the final weight of the same film after being kept in a desiccator with silica gel for 24 hours at room temperature (Arora \& Mukherjee, 2002).

$\%=\frac{m_{1}-m_{2}}{m_{2}} 100$

Percentage moisture uptake was calculated using Equation (2), where $m_{1}$ is the initial weight of a film and $m_{2}$ is the final weight of the same film after being kept in a desiccator with a saturated solution of sodium chloride instead of potassium chloride for $\mathbf{2 4}$ hours at room temperature (Arora \& Mukherjee, 2002).

$\%=\frac{m_{2}-m_{1}}{m_{1}} 100$

Dissolution test was carried out using a paddle apparatus (50 rpm) with the addition of a modified disc assembly in acetate buffer with $\mathrm{pH} 4.5$ tempered at $32{ }^{\circ} \mathrm{C} \pm 0.5^{\circ} \mathrm{C}$ (Ph. Eur. 10.4 , 2021). Fourteen samples were collected for 24 hours (SR8 Plus; Hanson Research, Los Angeles, CA, USA).

\section{RESULTS AND DISCUSSION}

Folding endurance was the main criterion taken into consideration when formulating the films. Firstly, it was noted that EC films require a plasticiser. Out of castor oil, polyethylene glycol and glycerine, the addition of $0.162 \mathrm{~g}$ of castor oil increased the number of folds to 100 . Moreover, the amount of EC was increased to $0.36 \mathrm{~g}$ to obtain more compact and flexible films. These films remained intact after 400 folds. For homogeneity of the films, it was necessary to direct the evaporating ethanol into a funnel during the drying process. The addition of 0.0225 or $0.045 \mathrm{~g}$ of HPMC caused $0.85 \%$ ( $p$ $=0.004)$ or $0.95 \%(p=0.002)$ increase in moisture content and $0.95 \%(p=0.278)$ and $1.93 \%(p=0.078)$ increase in moisture uptake, respectively. In our case, similar to what Dey et al. (2009) had mentioned, HPMC as a hydrophilic polymer increased the water content. HA addition did not cause a statistically significant difference in either moisture content or uptake.

We monitored the amount of propranolol released by dissolution in acetate buffer of $\mathrm{pH} 4.5$ for 24 hours and calculated the similarity $\left(f_{2}\right)$ and difference $\left(f_{1}\right)$ factors to compare the dissolution profiles. Of the total amount of propranolol in the samples, $28.41 \% \pm 3.30 \%$ was released from the EC film after 24 hours, the addition of HA $20.94 \%$ $\pm 1.52 \%\left(f_{1}=61.82 \pm 7.70, f_{2}=53.61 \pm 4.25\right)$ (Fig. 1) or HPMC $36.05 \% \pm 6.18 \%\left(f_{1}=34.48 \pm 8.79, f_{2}=65.02 \pm 5.33\right)$ (Fig. 2$)$. The dissolution profiles of $\mathrm{Ha} 1$ and HPMC1 films were compared with each other $\left(f_{1}=51.35 \pm 12.56, f_{2}=59.20 \pm 9.43\right)$. The Food and Drug Administration and the European Medicines Agency consider the dissolution profiles to be similar if $f_{1}$ is less than 15 and $f_{2}$ is greater than 50 (Costa \& Sousa Lobo, 2001). All similarity factors of the dissolution profiles of the films were above 50, and also, all difference factors were considerably above 15; we can therefore conclude that these profiles are not similar.

According to the coefficient of determination $\left(R_{2}\right)$, the drug followed Higuchi model in case of HPMC1 film $\left(R_{2}=0.9805 \pm\right.$ 0.0057) and Korsmeyer-Peppas model in case of Ha1 film $\left(R_{2}=\right.$ $0.9715 \pm 0.0032$ ). Release exponents (n) of Ha and HPMC films were in the range $0.5<\mathrm{n}<1.0$, which indicates non-Fickian diffusion of the drug. Based on the percentage of propranolol released from the formulations and their dissolution profiles, it can be stated that HA and HPMC are release rate modifiers in the prepared films. As Dey et al. (2009) had stated, the introduction of hydrophilic HPMC increased the dissolution rate in the case of water-soluble propranolol. However, such small amount of HPMC did not cause the initial burst effect unlike the case of the mentioned paper. HA is known for drug loading of cationic compounds and providing them prolonged release, possibly reducing the burst effect (Levis \& Deasy, 2003). Both of these effects are demonstrated in Fig. 1. In conclusion, we have prepared matrix-type propranolol patches without adhesives, which, to our knowledge, is novel in terms of composition. We have confirmed the possibility of alteration of the dissolution rate with the use of excipients such as HPMC for increasing and HA for decreasing the 


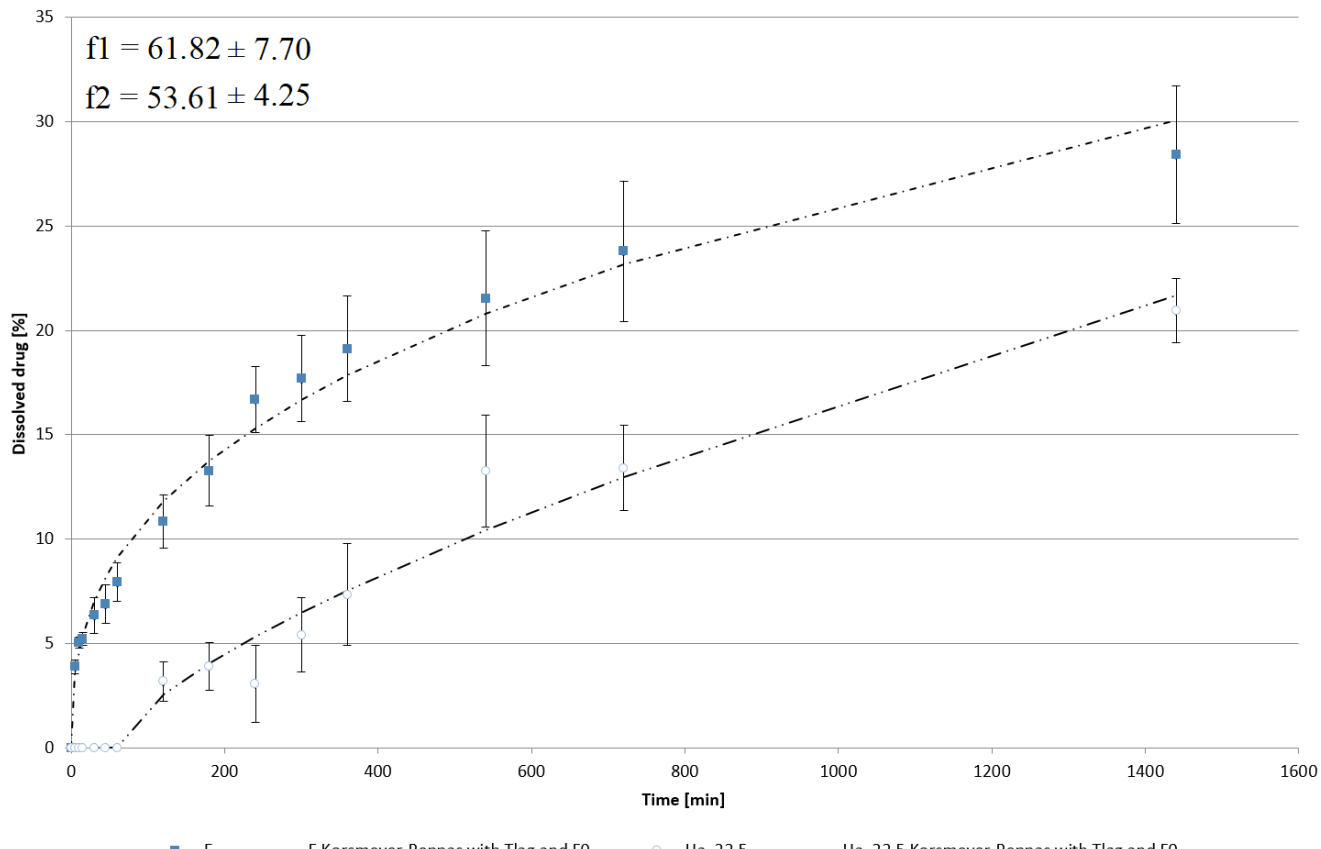

Figure 1. Dissolution profiles of formulations $\mathrm{E}$ and $\mathrm{Ha} 1$ compared.

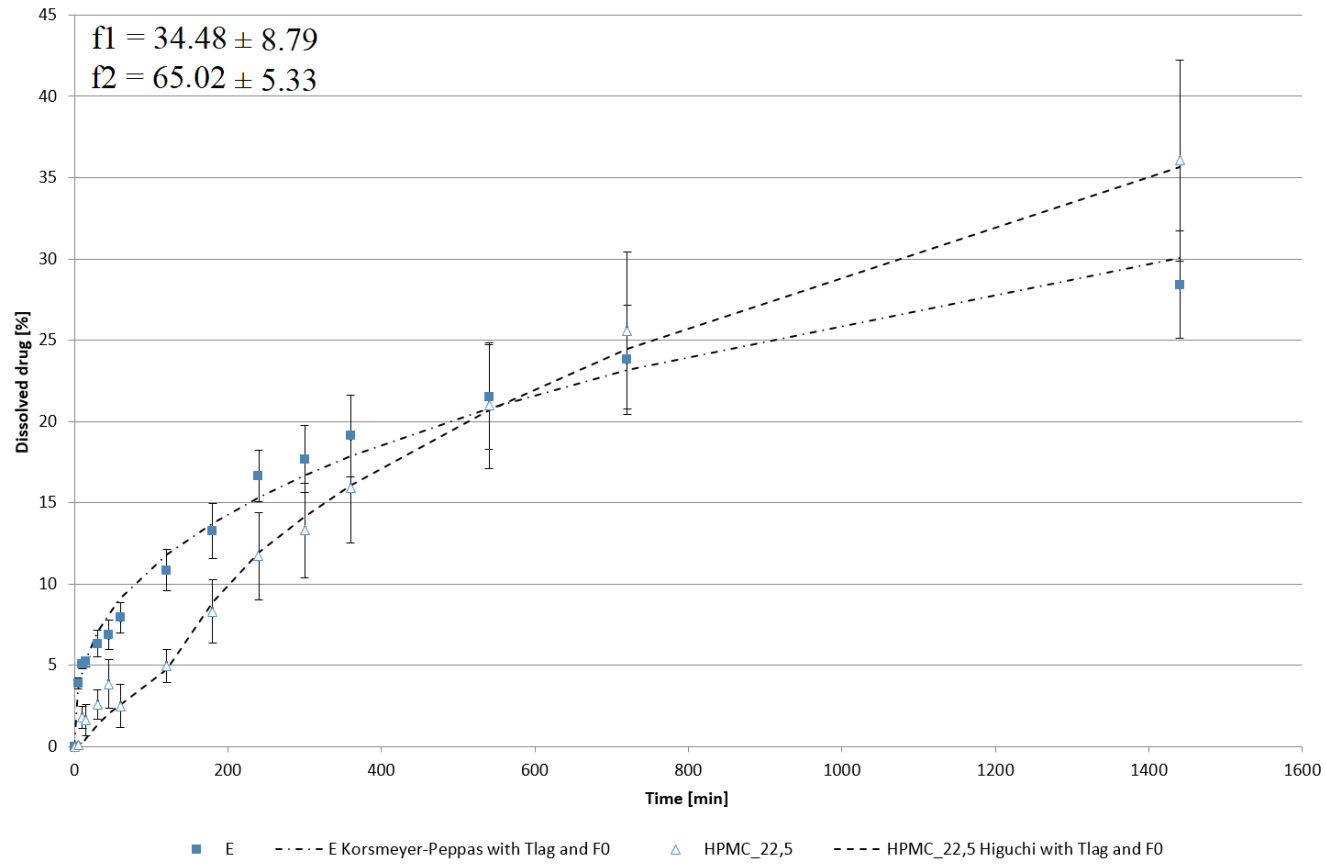

Figure 2. Dissolution profiles of formulations E and HPMC1 compared.

dissolution rate. However, since the daily dose of propranolol usually starts at $40-80 \mathrm{mg}$, the enhancement of dissolution rate should be considered keeping in mind the possible burst effect of hydrophilic polymers.

\section{ACKNOWLEDGEMENT}

The work was supported by the project from the structural funds 'Renewal of research and development infrastructure and instrumentation at UVLF, ITMS 26210120028'. 


\section{Transdermal Patches For Delivery of Beta-Blockers}

References

[1] Ahad A, Al-Jenoobi Fl, Al-Mohizea AM, Akhtar N, Raish M, Aqil M. Systemic delivery of $\beta$-blockers via transdermal route for hypertension. Saudi Pharm J. 2015;23(6):587-602.

[2] Aqil M, Sultana Y, Ali A. Transdermal delivery of $\beta$-blockers. Expert Opin Drug Deliv. 2006;3(3):405-18.

[3] Arora P, Mukherjee B. Design, development, physicochemical, and in vitro and in vivo evaluation of transdermal patches containing diclofenac diethylammonium salt. J Pharm Sci. 2002;91(9):2076-89.

[4] Calatayud-Pascual MA, Sebastian-Morelló M, BalaguerFernández C, Delgado-Charro MB, López-Castellano A, Merino $V$. Influence of chemical enhancers and iontophoresis on the in vitro transdermal permeation of propranolol: Evaluation by dermatopharmacokinetics. Pharmaceutics. 2018;10(4).

[5] Costa P, Sousa Lobo JM. Modeling and Comparison of Dissolution Profiles. Eur J Pharm Sci. 2001;13(2):123-133.

[6] Dey BK, Kar PK, Nath LK. Formulation design, preparation and in vitro - in vivo evaluation of propranolol hydrochloride transdermal patches using hydrophilic and hydrophobic polymer complex. Research J. Pharm. and Tech. 2009;2(1):155-160.

[7] European pharmacopoeia 10.4. 2.9.4. Dissolution test for transdermal patches. (https://pheur.edqm.eu/app/10-4/ content/10-4/20904E.htm?highlight=on\&terms=dissolution). Revised April 2021. Accessed June 7, 2021.

[8] Levis SR, Deasy PB. Use of coated microtubular halloysite for the sustained release of diltiazem hydrochloride and propranolol hydrochloride. Int J Pharm. 2003;253(1-2):145-57.

[9] Sanap GS, Dama GY, Hande AS et al. Preparation of transdermal monolithic systems of indapamide by solvent casting method and the use of vegetable oils as permeation enhancer. Int J Green Pharm. 2008;2(2):129. 